their relations to normal animals, renders it probable that its further application will throw light on the conditions underlying the association of the inception of cancer with the senescence of tissues, and on the increasing frequency of the disease as age advances throughout the vertebrate kingdom.

\title{
A SPECIFIC LUNG DISEASE OF CALVES.
}

By LL. E. W. Bevan, M.R.C.V.S., Government Veterinary Surgeon, Salisbury, Rhodesia.

FROM time to time during the past eight years a lung disease of calves has been prevalent on certain dairy farms in the neighbourhood of Salisbury, Rhodesia. The mortality from the disease has been very serious. In I9OO, at one dairy, thirty-five calves died out of seventy; in I90I seventy out of ninety succumbed on this dairy, and a second dairy suffered in an equally serious manner.

Dormant during 1903 and I9O4, the years when African coast fever accounted for most of the mortalities among young stock, it again appeared in 1905 .

Fortunately the last outbreaks have not been accompanied by such a heavy death-rate-a result partly due to therapeutic measures adopted, but chiefly attributable to the more rigorous prophylactic measures instituted by the Veterinary Department and by the owners themselves, who have learnt the extreme gravity of the disease and the necessity for hygienic precautions.

It is possible that the disease exists in other parts of the country, but it is only in Salisbury that the malady has taken on a purely pneumonic character. In Matabeleland reports of what is called "liver disease" among dairy calves are frequently recorded, but it would appear that this is distinct from the lung disease about to be described, although means employed to combat the latter have been said to have been followed by beneficial results when adopted to the liver complaint. A possible explanation for this may be found in the following notes.

Occurrence.-During the first outbreaks of the disease it was thought that it occurred only among calves between the ages of six weeks and six months; but, although animals of this age would appear to be the principal victims, it has been found that cases do occur, in exceptional circumstances, in calves as old as nine months. The disease would appear to be one of certain localities, for during the fast few outbreaks it has been possible to incriminate certain small areas; indeed, at one dairy, a small calf-kraal, about 20 feet by io feet, was found, by a process of exclusion, to be the principal site of the infection.

If the disease is allowed to run unchecked, and the calves to wander indiscriminately over the dairy farm, the whole locality becomes contaminated, and one dairyman was compelled to move his dairy twice on this account. The third attempt was followed by a similar unfortunate state of affairs, and the system of sending in-calf cows to an outlying farm to calve had to be adopted. 
The change from the second to the third site, in the particular instance, was attended by a very interesting result, which may be alluded to here. The disease had made its appearance at the second dairy in 1905, but, owing to the severe methods of isolation and disinfection practised, was "got in hand" with but a small death-rate. The whole dairy and every animal on it was so frequently and thoroughly sprayed with strong disinfectants that it appeared, from the cessation of the disease, that the infection had been destroyed. It may be mentioned that at that time and during the following year the law forbade young calves being allowed to run at large on areas where African coast fever had existed within the previous twelve months, and Salisbury Commonage being one of these infected areas, the calves at the dairy in question were continually kept enclosed in a calf-pen, and were systematically dipped.

In February 1907 it was found necessary to enlarge the dairy, and certain fittings were removed to a new establishment. Calves born at this time soon after birth developed unmistakable symptoms of the lung disease, and it was at the time concluded that the dismantling of the buildings of the second dairy had liberated or exposed the causal organism of the disease.

At the time of the changing of the second dairy to the new site, Salisbury Commonage was declared free from danger from African coast fever, and the quarantine imposed upon calves was raised. Bovine malaria (Texas fever or redwater) being endemic to the Salisbury district, these calves were thus for the first time exposed to gross infection by the blue tick, which was plentiful at the time of their release, and, as a result, they suffered from piroplasmosis in a greater or lesser degree, some dying from acute hæmoglobinuria. It was during this particular outbreak noticed for the first time that calves as old as nine months were liable to suffer from the lung complaint.

The possible connection between redwater and the lung disease will be discussed later, but it may be further recorded in this place that during the past months the disease has appeared on a dairy farm free from the malady since I9OI, and that the sick calves have been covered with the blue tick, which has become abundant with the first rains. Examination of blood smears from the calves on this farm was not made until the disease had established itself, but has been latterly carried out, with the result that the blood of several of the calves suffering from the lung disease has shown lesions of piroplasmosis-either parasitic invasion of red cells, or alterations such as poikilocytosis and basophile granulations. Further, the blood of four calves which, by way of experiment, had been housed apart in a hut since birth, and were free from lung disease, was apparently normal.

The disease generally occurs during the early months of the year, during the rainy season; but on more than one occasion it has appeared in October and November, before the rains have commenced. After the first case has made its appearance others follow in alarming rapidity, and it is not long before nearly every calf on the farm is suffering more or less severely. This particularly relates to calves which are confined to a small area; calves running constantly with their mothers have been known to escape, although coming into contact with sick calves when brought up at milking time. When 
an outbreak has become fairly established, however, it is rare that any of the calves entirely escape.

Symptoms. - The affected calves show the usual symptoms of ill health, namely, staring coat, dry muzzle, tucked-up and unthrifty appearance. A sick calf may be detected early in the disease by the listless manner in which it stands and by the dry and staring condition of its coat; later, the skin becomes scurfy and wrinkled, and appears particularly liable to invasion by parasites, especially ticks. After the disease has progressed for a day or two the calf moves unsteadily, or lies about breathing heavily and even panting, with its mouth open and its tongue protruding.

Among the younger animals a foetid diarrhœe has been marked, the faces being of a light colour, sometimes clay-coloured or slatecoloured, and occasionally resembling the fæces met with in specific white scour. In one or two cases dysentery has been present. The dung generally has a very offensive odour, and accumulates around the animal's hind quarters, rendering the calf and the place in which it is quartered filthy in the extreme.

During an outbreak cases have been met with in which the scouring has not been accompanied by lung symptoms; and it may be mentioned that a recent outbreak of specific white scour has proved that that disease, per se, does exist in this district.

Perhaps the most characteristic symptom of the disease is a short cough, chiefly noticed when the animal is disturbed or when it first leaves the shed and comes into the cooler air outside, or when it first takes milk from its mother. It was by this well-known symptom that the disease was found to be present in those older calves which did not manifest the usual attendant symptoms in such a marked degree. As the disease progresses the breath of the sick calves often becomes offensive, and in a few cases an ulcerated condition of the tongue and mucous membranes of the mouth has been observed. In one or two instances an accumulation of pus has been met with at the umbilicus, causing a large and visible swelling noticeable during life. In not a few cases, where the disease has been complicated with redwater, hæmoglobinuria has been present.

A remarkable feature of the disease is the fact that the sick animal does not refuse its food, and that calves not weaned will suck their mothers as long as they have the strength to do so. In those cases among older callves the lung disease has alvays been aggravated by redwater, and there has been some loss of appetite, attributable rather to the latter disease than to the lung affection.

Lesions.-In the majority of cases, on post-mortem examination, the principal lesions are met with in the lungs, which are found studded with numerous small nodules, varying from the size of a millet grain to that of a pea. In the early stages of the disease these nodules may not appear on the surface of the lung, and their presence may only be detected by palpation. Later, they are visible as small elevations on the surface of the lung, and resemble in many respects a tubercle nodule. In a well advanced case the nodules become densely clustered together, and may render portions of the lung almost solid. In addition to this nodulated condition there is generally present a certain amount of ordinary pneumonia, and occasionally pleurisy and adhesions are met with. On section, the 
nodules may be shelled out, and are found to be made up of a cheesy material of a yellow colour and again recalling a tubercle nodule, except that calcification does not appear to take place. The anterior lobes of the lung seem particularly liable to become involved, especially at their edges. In some cases abscesses are found in the liver, and on one or two occasions they have been met with at the navel. In those cases where scour has been marked during life an inflammatory condition of the bowels may be observed. In a large number of instances it has been remarked that, on setting, the fat of the carcase has become a bright yellow colour, and the liver, after exposure to the air for a few minutes, has become a bright ochre colour. The kidneys also have become yellow in a similar manner. These were no doubt cases complicated with redwater.

Differential Diagnosis. - The Rhodesian dairy calf disease appears to differ in many respects from other calf diseases described in recent literature. To some extent it recalls the disease investigated in I90 I by Professor Nocard ${ }^{1}$ in the south-western parts of Ireland, but the following table shows the various points of difference :-

\section{White Scour (Nocard).}

Manifests itself by diarrhoea, which usually appears on the second or third day following birth, rarely it makes its appearance on the first day, sometimes as late as the sixth. If the calf shows no signs after the eighth day the farmer thinks his calf will escape (Mettam).

The calf refuses its food.

In some cases of acute white scour there is intense arthritis of one or more joints.

Principal post-mortem lesions found in the abdominal cavity.

The lungs show areas of collapse, often small lesions of hæmorrhagic pneumonia (Mettam).

Causal organism said to be one of the fowl cholera type.

\section{Rhodesian Disease.}

Diarrhoea not a constant feature. Usually appears between the sixth week and the sixth month.
In the thoracic cavity.

Typical nodules.

The septicæmia of calves in the neighbourhood of Utrecht, described by Thommasen, ${ }^{2}$ bears features of resemblance to the Rhodesian disease, the following being the principal :-

\footnotetext{
1 Paper read by Professor Mettam at the meeting of the National Veterinary Association at Windermere, on 10th September 1903.

2 “Annales de l'Institut. Pasteur," Tone. XI., p. 523, 1897.
} 
"The muzzle is dry and the respirations hurried; sometimes there is a dry cough. Though the appetite is not good, still the animal will take milk. Diarrhœa has been noticed."

The diseases differ, however, in the following features:-

\section{Thommasen's Disease of Utrecht Calves.}

Principal lesions nephritis and urocystitis.

First symptoms observed on the fifth to eighth day, and even as late as four to five weeks.

When made to get up, calves stretch themselves and bend downwards the back and loins.

\footnotetext{
The thoracic organs were sound.
}

\section{Rhodesian Disease.}

Kidneys and bladder not affected in cases not accompanied by redwater.

Symptoms usually delayed till sixth week.

Arch upwards the back and loins.

Lesions chiefly found in thoracic organs.

The disease known as "septic pleuro-pneumonia," described by Jensen and Poels, and prevalent among calves in Holland, Belgium, Denmark, Prussia, France, Italy, and Russia, is similar to the Rhodesian disease in that the principal lesions are within the chest: "Lung symptoms make their appearance, as noted by the respirations and cough, and by the soreness of the chest to palpation and manipulation generally. A fœtid diarrhœa then sets in."

In Jensen's disease, also, there is reason to believe that it is spread by infection and contagion, and it is possible that the causal organism is a facultative parasite present in certain soils and upon forage, obtaining entrance into the body by the respiratory tract, or by the alimentary tract, and in the case of the Salisbury disease by way of the umbilicus at the time of, or shortly after, birth. Jensen's disease, however, differs from the disease under discussion in the following respects :-

\section{Jensen's Pleuro-Pneumonia of Calves.}

Also affects pigs and kids.

Often the subject dies in a few hours after. it is noticed to be ill.

The lung is increased in size, hard, and friable, and in appearance is likened to the lesions of contagious pleuro-pneumonia.

\section{Rhodesian Disease.}

Observed in calves only.

Progress comparatively slow; duration about a week in fatal cases.

Characteristic nodules. (In exceptional cases a resemblance to contagious pleuro-pneumonia has been remarked.)

In the September issue, 1907, of the Agricultural Journal of the Cape of Good Hope, an article by $\mathrm{Mr}$ William Robertson appeared 
under the heading of "Pneumo-Enteritis, or Pasteurella Bovis: A Stock Disease of the Eastern Coastal Districts, sometimes confounded with Lamziekte, or Osteomalacia." Accompanying the article were four photographs of calves presenting appearances almost identical with those met with in calves suffering from the Salisbury disease. Another photograph of the lungs of a sheep killed by intravenous inoculation of a culture from an ox, showing the distension of interlobular spaces with straw-coloured oedema, recalled a condition not unfrequently accompanying the nodular affection of the lungs in Salisbury calves.

A description of a typical outbreak of the calf disease of the Eastern coastal districts coincides in many respects with outbreaks of the Salisbury malady, but the following points would indicate that the maladies are distinct :-

\section{Disease of Calves in Eastern Coastal Districts of Cape Colony (Robertson).}

Edema of lungs.

Total absence of fat in the carcase.

Abdominal lesions.

Causal organism (a bacillus) isolated.

\section{Rhodesian Disease.}

Typical lung nodules; œdema only occasionally.

Fat scarce, but not entirely absent.

Abdominal viscera generally healthy.

Attempts to isolate causal organism not successful.

Predisposing Infuences.-In Professor A. E. Mettam's paper "On certain Septicæmias and some other Infections of Young Animals," read at the meeting of the National Veterinary Association, held at Windermere on Ioth September I903, the following statement occurs : "It appears that anything which will injure the natural defences of the body will allow of the entrance of organisms which may produce death, and that these organisms are generally harmless when in the lumen of the bowel. . . . There is also considerable evidence in support of the contention that secondary lesions of a septicæmic from which the animals recover are due to the entrance into the body of organisms which are normal inhabitants of the intestines, and which, under ordinary circumstances, thrive there without doing harm to the host. Indeed, it is probable that many members of the intestinal flora are absolutely essential to thorough and efficient digestion in the intestinal tube, and to the normal development of the host. . . . Still, from the knowledge we have already gained as to the life-history of organisms, we are driven to conclude that, from some cause or condition or other, parasites or saprophytes which are ordinarily harmless may produce varieties which may give rise to serious lesions."

Following this supposition, it might be suggested that calves born on the scene of previous outbreaks of the disease under consideration admit into the body an organism which, in ordinary circumstances, 
produces no pathogenic effects, but that, given some conditions capable of injuring or weakening the natural defences of the body, this specific organism is no longer held in check, and is then capable of exerting its noxious properties.

The manner in which the organism gains entrance into the body has not yet been determined, but there is reason to believe that the chief means of infection is through the uncicatrised umbilicus. The principal evidence in favour of this supposition is the favourable results which have followed careful attention to the umbilicus at the time of birth. Further, in a few cases, abscesses have been met with in this situation.

In a report to the Chief Veterinary Surgeon concerning the Igo6 outbreak, the following statement occurs: "Of the forty calves there is not one which can safely be described as unaffected, but I would draw your attention to the fact that there are two calves which have not shown symptoms of the disease up to the present, and these are so obviously in better health than their fellows that the cattle inspector was able, without trouble, to point them out to me when asked to do so as a test. These animals, having been born on muddy days, have had their navel-strings ligatured, and, on the owner's own admission, these two only have received this treatment."

Up to the present, however, there is no evidence to exclude the other possible methods of infection; and, since the sick calf contaminates its surroundings with discharges from its nose and mouth and with its dung, there would appear to be every possibility of organisms being inhaled or ingested. The chief evidence in favour of the theory that the organism is admitted into the body and remains there without producing pathogenic effects is met with in those rare cases where the animals have escaped the disease during the period of life when it usually makes its appearance (i.e., from six weeks to six months), but have developed symptoms when as old as nine months when the victims of redwater.

Further evidence in favour of this theory lies in the following incident. A dairyman, on whose dairy the disease, was rampant, particularly wished to save two calves from valuable Jersey cows, and a few days after birth removed them to a farm a few miles from town, where the disease had never occurred. These calves were carefully housed and tended, but six weeks after removal one died with typical symptoms of the disease. Its lungs were studded with the characteristic nodules.

The circumstances which tend to reduce the natural defences of the calf may be one or more of the following :-

(I) Inclement weather. The disease, as a rule, appears during the months of January, February, and March, when the rains are at their worst. The last outbreak occurred during October, just before the rainy season set in, when the calves suffered from the intense heat. At this time, too, there was very little grazing for the cows, and the calves suffered from want of milk.

(2) Dietetic errors. In this country the dairyman has to contend with a great many difficulties in feeding his calves. In the first place, his cows are only average milkers, and if the calf takes as much milk as is really necessary for its development there is little over for dairy purposes. The custom therefore is to allow the calf to take, 
morning and evening, as much as is left after the cow has been milked. Attempts have been made to foster the calves from the milch cows upon inferior cows or those used for breeding beef or trek animals. This is not always possible, and, in addition, it is generally held that in this country a cow will not "drop her milk" without her calf. The idea has always been met with ridicule by experts, but it would appear to be true in the case of badly-bred or native cows which have previously suckled a calf. Moreover, the native milkers, prompted by superstition or laziness, if set to milk a calfless cow do so in a half-hearted manner, and the yield of milk quickly ceases. Again, in Rhodesia, the prohibitive prices of codliver oil, linseed, calf-meal, and other fat substitutes render artificial feeding extremely expensive if not impracticable.

(3) A low state of health, the result of keeping animals penned up in small and insanitary calf-kraals, often devoid of a roof, and knee-deep in mud.

(4) Redwater. As has been said before, bovine malaria is endemic to the Salisbury district, and every calf born and allowed to run in this district is subject to redwater infection from the time of birth. That this is so has been amply proved by the results following the inoculation of newly imported cattle arriving from non-redwater areas of the Cape Colony with blood from Commonage calves never noticed to be sick. Several of such inoculated animals have died, and piroplasmosis has been evident on microscopic examination of the blood of every animal so treated.

During the past two years the association between the lung sickness and redwater has been very marked. It was especially noticeable during the I906 outbreak, when attention was drawn to the fact by the occurrence of severe hæmoglobinuria in several of the calves suffering from the lung disease. This is the outbreak previously referred to as occurring among calves newly liberated and allowed to run on the Commonage after months of confinement in a small kraal on the dairy premises. It should be particularly mentioned that during this time these calves were systematically dipped,-a very important feature, since kraaled animals are liable to become almost as badly infested with ticks as animals allowed to run. Experience of African coast fever has demonstrated how a tick-borne disease will linger in kraals and calf-pens longer than in other places, no doubt because ticks find therein exceptionally favourable opportunities for engorging and hiding during the progress of their life cycle. Possibly, therefore, the calves in question, being submitted to a sudden gross infection, suffered more severely from redwater than animals constantly exposed from birth, which contract the disease gradually without showing signs of sickness.

The exceptional occurrence of the lung disease in nine-months-old calves, simultaneously with acute and newly contracted piroplasmosis, suggested a very close association between the two diseases.

Treatment.-The treatment of this disease has offered exceptional difficulties, by reason of the numerous complications with which it is generally associated, so that it has been found necessary to first treat those symptoms which predominate in each case. For example, where a calf shows marked symptoms of redwater it has been advisable to administer a solution of $500 \mathrm{cgm}$. of methyldinatrium- 
arsenate dissolved in $5 \mathrm{cc}$. of distilled water. Experience has proved this agent to be particularly valuable in both redwater and equine piroplasmosis. The solution is injected once daily for two or three days, direct into the jugular vein - a method well tolerated, and having the advantage of avoiding the very serious abscesses and sloughs which often follow the subcutaneous exhibition of the drug.

Cases complicated by diarrhœea and dysentery have been treated with an emulsion composed of a purgative, antacid, and disinfectant. Creosote in five-minim doses has been found a valuable intestinal antiseptic in such cases.

Of the many agents tried in the treatment of the lung disease itself, a proprietary article known as "Tallianine" has been followed by the best results. This drug is said to be obtained by the action of ozone upon a terpene-bearing volatile oil, the process being stopped at a point when the resulting product is capable of evolving four times its own volume of ozone. This product is administered by intravenous injection, usually into the jugular, and is said to provoke an abundant leucocytosis.

In dealing with all outbreaks, the first endeavour has been to locate the infected area and to submit it to most vigorous disinfection. All the fittings and animals on the dairy have been carefully disinfected, while the cows and calves have been regularly dipped or sprayed with arsenical dips. A few drops of izal have been regularly added to the water in the drinking troughs, and it has been found that when once the animals become accustomed to it they show no disinclination to drink.

Dairymen have been carefully instructed in the usual hygienic precautions necessary for the maintenance of health, not only of their stock, but also of the consumers of the milk, but it must be admitted that these precautions are very incompletely carried out. The washing of the cow's udders with a disinfectant before allowing the calves to suck and the disinfection and ligaturing of the navel at the time of birth have been recommended, and when conscientiously carried out have been followed by a very satisfactory reduction in the number of cases.

With a class of somewhat indifferent and self-satisfied dairymen to deal with, with filthy and ill-constructed dairies to work in, and with native labour to depend upon, it is not surprising that the efforts of the Veterinary Department have been attended with only partial success.

\section{SPIROCH ETOSIS OF FOWLS IN SOUTHERN RHODESIA.}

By LL. E. W. Bevan, M.R.C.V.S., Government Veterinary Surgeon, Salisbury, Rhodesia.

WITHIN the last few years a very serious disease of chickens has made its appearance in and around the townships of Southern Rhodesia, and has proved a very serious handicap to the poultry industry. While experience has shown that the disease is in some way associated with the common fowl tick (argas persicus), the 